\title{
Screen printing tissue models using chemically crosslinked hydrogel systems: a simple approach to efficiently make highly tunable matrices
}

\author{
Narendra Pandala ${ }^{1}$, Michael A. LaScola ${ }^{1}$, Yanchun Tang ${ }^{2}$, Maria Bieberich ${ }^{1}$, LaShanda T.J. \\ Korley $^{23}$, Erin Lavik ${ }^{1^{*}}$
}

${ }^{1}$ Chemical, Biochemical and Environmental Engineering, University of Maryland Baltimore County, Baltimore, Maryland 21250, Piscataway Territories, United States

${ }^{2}$ Department of Material Sciences and Engineering, University of Delaware, Newark, Delaware 19716, United States

${ }^{3}$ Department of Chemical and Biomolecular Engineering, University of Delaware, Newark, Delaware 19716, United States

\section{Corresponding Author}

* Erin Lavik

Email: $\underline{\text { elavik@umbc.edu }}$

No. of pages -13 
No. of figures - 11

\section{SUPPLEMENTARY INFORMATION}

\section{EXPERIMENTAL SECTION}

\section{Materials}

4arm PEG-Succinimidyl Gluterate(10kDa) was purchased from Jenkem, USA. Linear PEG(4.6kDa), 1,1'-Carbonyldiimidazole (CDI), 1,4-Dioxane, Poly-L-Lysine (PLL), N,N Disuccinimidyl carbonate, 4 dimethyl amino pyridine, Anti-Laminin antibody produced in rabbit were purchased from Sigma. Goat anti-Rabbit IgG (H+L) Cross-Adsorbed Secondary Antibody, acetone, diethyl ether and other solvents, media components (MEM alpha and DMEM), Fetal Bovine Serum, HEPES, Sodium pyruvate, Live dead stain, Dil and Dio stain, dialysis tubing were purchased from ThermoFischer/Fischer Scientific. PEG thiol was purchased from Laysan Bio. Divinyl sulfone (DVS) from Alfa Aeasar. and MTT assay kit from abcam. Fluoresbrite BB carboxylate Microspheres $(0.10 \mu \mathrm{m})$ were purchased from Histo-Line Labs (Italy). Caco-2 cells were obtained from ATCC, USA.

\section{Methods}

\section{PEG activation by CDI}

Linear dihydroxyl Polyethylene Glycol (PEG) (mol wt. $4.6 \mathrm{kDa}$ ) is dissolved in 1,4-Dioxane at $37^{\circ} \mathrm{C}$ to a concentration of $0.05 \mathrm{gm} / \mathrm{ml}$ and $1: 1$ molar ratio of 1,1'-Carbonyldiimidazole (CDI) to PEG is added. The mixture is stirred for 2 hours at $37^{\circ} \mathrm{C}$. The unreacted CDI and the dioxane in 
the reaction mixture are then removed by dialysis in deionized water (MWCO- $1 \mathrm{kDa})$ at room temperature. The resulting mixture is flash frozen in liquid nitrogen and lyophilized for 2 days.

\section{VS-PEG-PLL synthesis}

$1 \mathrm{gm}$ of PEG imidazole carbamate active intermediate synthesized is dissolved in $50 \mathrm{ml}$ of $50 \mathrm{mM}$ Sodium bicarbonate (pH=8.2). 250mg of Poly-L-Lysine (PLL) (mol wt. $110 \mathrm{kDa}$ ) dissolved in 5 $\mathrm{ml}$ of 50mM Sodium Bicarbonate $(\mathrm{pH}=8.2)$. The PEG carbamate solution is added dropwise to the PLL solution and stirred for 2 hours at room temperature. The $\mathrm{pH}$ of the above solution is raised to 13 and $0.750 \mathrm{ml}$ of divinyl sulfone (DVS) is added to the above mixture and stirred for 10 minutes at room temperature. The reaction is stopped by lowering the $\mathrm{pH}$ of the mixture below 5 and the unreacted vinyl sulfone, PEG and other components are removed by dialysis in deionized water (MWCO- 50kDa) at room temperature overnight. The solution is flash frozen in liquid nitrogen and lyophilized for 2 days.

\section{PEG-SG synthesis}

4.6gms of dihydroxyl PEG(1mmol) is dissolved in $25 \mathrm{ml}$ of dry dioxane, $3.1 \mathrm{gms}$ of $\mathrm{N}, \mathrm{N}$ Disuccinimidyl carbonate $(12 \mathrm{mmol})$ is dissolved in $40 \mathrm{ml}$ of dry acetone and the $1.5 \mathrm{gms}$ of 4 dimethyl amino pyridine $(12 \mathrm{mmol})$ is dissolved in $20 \mathrm{ml}$ of dry acetone. The three solutions are mixed and stirred for six hours at room temperature, then the product is precipitated using diethyl ether and the precipitate is dried, the precipitate is dissolved in deionized water and dialyzed for one hour at room temperature using MWCO-1kDa tubing

\section{Hydrogel synthesis}

VS-SH system: $10 \% \mathrm{wt} . / \mathrm{vol}$. solutions of VS-PEG-PLL macromer obtained from the above steps, and commercially available dithiol PEG (SH-PEG-SH) (mol wt. 3.4kDa) are prepared in 0.3 M triethanolamine $(\mathrm{pH}=8)$ solution. The above two solutions are warmed to $37^{\circ} \mathrm{C}$ and mixed in $1: 1$ to molar ratio of VS to $\mathrm{SH}$. The solution is kept at $37^{\circ} \mathrm{C}$ until it is completely gelled. 
$\underline{\text { SG-NH}} 2$ system: $10 \%$ w/vol. solution of PEG-SG and the PLL solution in PBS (for Rheometry) and the culture media (for the cells) and mixed according to the ratio of free amines to succinimides and then set it at 37C until completely gelled. For the hydrogel supplemented with Laminin, $100 \mathrm{ug} / \mathrm{ml}$ of Laminin is added to the PLL solution and incubated for an hour at $37^{\circ} \mathrm{C}$ before mixing the PLL and the PEG solutions.

For seeding the cells, on top of the hydrogel base, the gel is washed (2x, 5 minutes) with media before adding the cells on the hydrogel base. For seeding the cells inside the hydrogel, the precursor solutions are mixed with the cells and the hydrogel is washed (2x, 5 minutes) with media.

\section{Rheology}

The storage and loss moduli and gelation time results were obtained using a Stress Control Rotational Shear Rheometer attached with a stainless steel 20-mm-diameter parallel plate geometry for the time sweep and $40 \mathrm{~mm}$ diameter parallel geometry stainless steel plate for the frequency sweep. For gelation time the gel precursor solution is mixed and immediately placed on the rheometer a time sweep was performed as describes in Zuidema et.al. at $37^{\circ} \mathrm{C}$ using a AR 2000 instrument (TA instruments) ${ }^{1}$. For the frequency sweeps a gel of 600ul was fabricated and completed set and a frequency sweep was performed at a compressive force of $0.5 \mathrm{~N}$ using a Discovery HR3 system (TA instruments).

\section{SEM characterization}

The hydrogel is synthesized and set to gel completely in a humid chamber at $37^{\circ} \mathrm{C}$ and then it is swollen in excess of water overnight at room temperature, the excess water is removed, and the swollen hydrogel is kept at $-20^{\circ} \mathrm{C}$ overnight and then flash frozen using liquid nitrogen and lyophilized for two days. The dried hydrogel is then coated with a $20 \mathrm{~nm}$ layer of gold palladium using a vacuum sputter coater (Denton Desk II) and then the hydrogel sample was imaged using a Nova NanoSEM 450(FEI). 


\section{NMR}

Approximately $10 \mathrm{mg} / \mathrm{ml}$ of the analyte solution is prepared in deuterated water and the NMR spectrum was measured using JEOL 400Mhz NMR system.

\section{OPA assay}

OPA assay was performed using the solutions of the synthesized VS-PEG-PLL macromer in PBS OPA reagent against a standard curve of Poly-L-Lysine according to the method described by Grotzky et. al. ${ }^{2}$

\section{Screen Printing}

Screens are constructed and sterilized according to the protocol described previously ${ }^{3}$. Gel precursor solutions, along with cells were mixed to a total concentration of $10 \% \mathrm{w} / \mathrm{vol}$ and placed near the stencil on the screen and passed onto a clean cover slip or a slide using an aluminum or a rubber squeegee. To analyze the quality of the prints obtained using PEG-PLL gels, florescent carboxylate microspheres $(0.10 \mu \mathrm{m})$ were mixed with the precursor solutions and different patterns were printed onto clean glass slides.

\section{Cell culture}

Caco-2 cells were cultured in MEM alpha supplemented with 20\% FBS. Rat endothelial cells were cultured in DMEM High Glucose supplemented with 10\% FBS, 1\% HEPES and 1\% Sodium pyruvate.

\section{Cell staining}

$4 \mu \mathrm{m}$ of calcien-AM and $8 \mu \mathrm{m}$ of ethidium homodimer were used to stain the live and dead cells respectively, the cells were imaged after 40 mins of incubation at $37^{\circ} \mathrm{C}$.

\section{Cellular viability}


Cellular viability of the cell laded hydrogels was obtained using the MTT assay kit (abcam) and the survival of the conditions was calculated according the formula. We used a serum free media without any phenol red for the hydrogel fabrication, and for the MTT assay. OD sample is the raw absorbance of the sample, $\mathrm{OD}_{\text {control }}$ is the absorbance of the cells seeded on the well plate with media and no hydrogel, the $\mathrm{OD}_{\text {blank }}$ is the average absorbance of the wells with media and wells with hydrogel without any cells.

percentage viability $=\left(O D_{\text {sample }}-O D_{\text {blank }}\right) /\left(O D_{\text {control }}-O D_{\text {blank }}\right)$

\section{Laminin Staining}

Hydrogels were prepared by mixing different volumes of PEG and PLL and the completely set hydrogels were embedded in the OCT freezing media. $20 \mathrm{um}$ sections of the gels were obtained at $-25^{\circ} \mathrm{C}$ and stained using Anti-Laminin antibody produced in rabbit as the primary antibody and the Goat anti-Rabbit IgG (H+L) Cross-Adsorbed Secondary Antibody, Texas Red as the secondary antibody.

\section{Imaging and Analysis}

The florescent imaging was performed using Cytation 5 (Biotek, USA) and the composite images were obtained using a z stack with 4 images at $2 \mu \mathrm{m}$ layers and a $15 \mathrm{X} 15$ grid of images on each $\mathrm{z}$ image, the z-stack is blended using a focus stacking and the $x-y$ images are stitched together by a linear blend using the Gen 5 imaging software (Biotek, USA). The heat maps and the bright field images of the prints are obtained using a laser scanning confocal microscope (VK-X1000, Keyance, Japan), and the composite images are stitched together using Adobe Photoshop. The pore size of the SEM micrographs was analyzed using ImageJ and the pore size is determined from the area obtained using the SEM. 


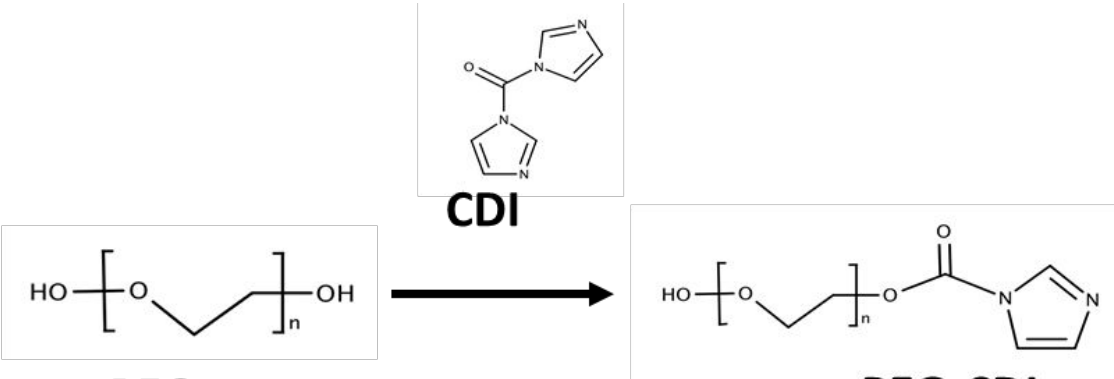

PEG

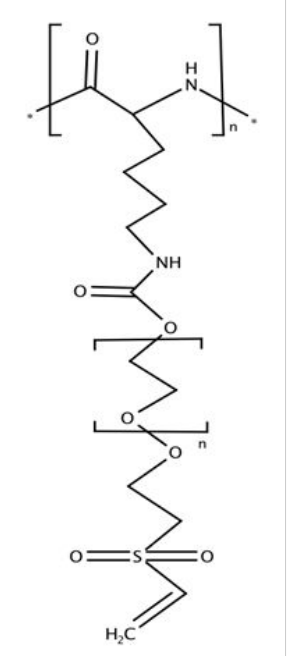

VS-PEG-PLL
PEG-CDI
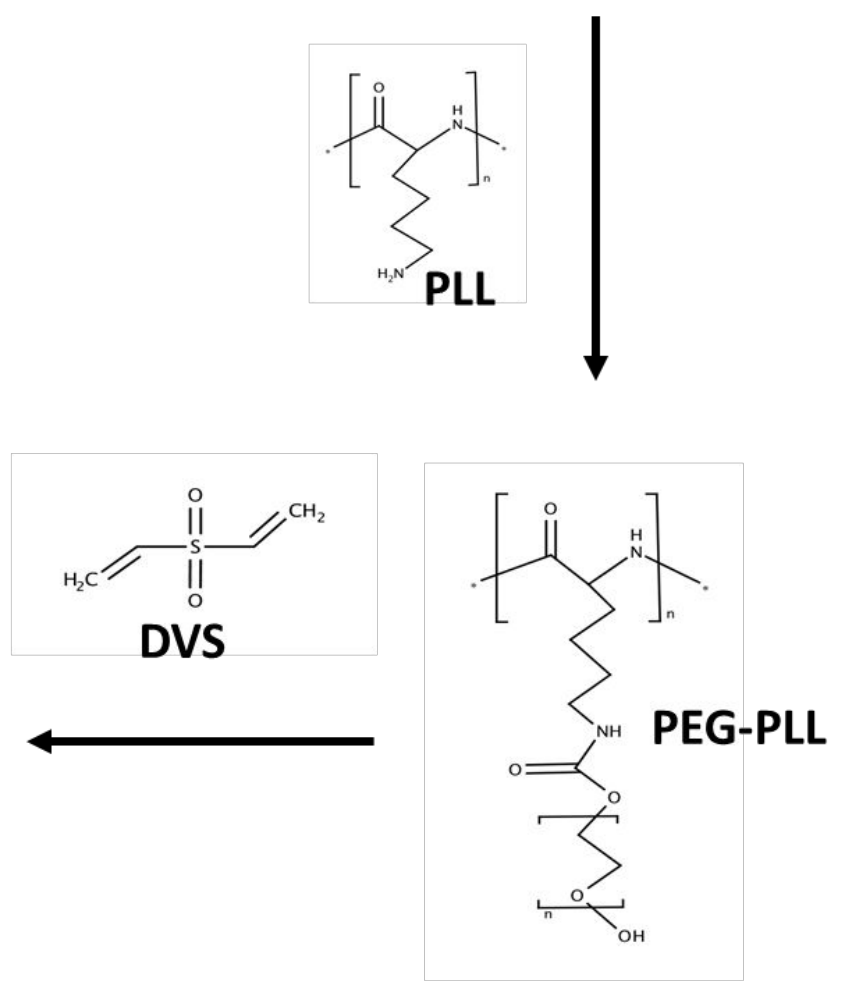

Figure S1. Synthesis of VS-PEG-PLL macromer, starting from the dihydroxyl PEG which turns into an intermediate PEG-CDI compound with the addition of CDI, which is then reacted with PLL to form PEG-PLL; later the hydrpxyl ends of PEG in the PEG-PLL compound formed are functionalized by using divinyl sulfone to form VS-PEG-PLL macromer 


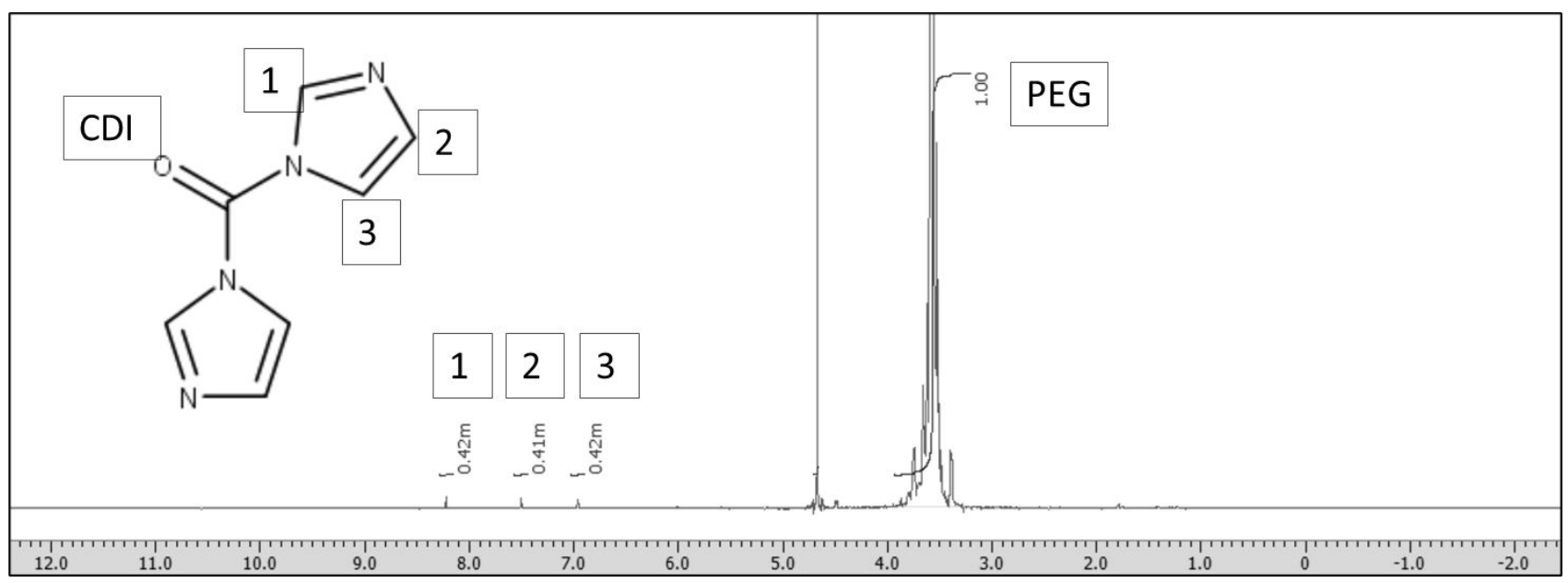

Figure S2. ${ }^{1} \mathrm{H} N \mathrm{NR}$ of the PEG-CDI intermediate formed.

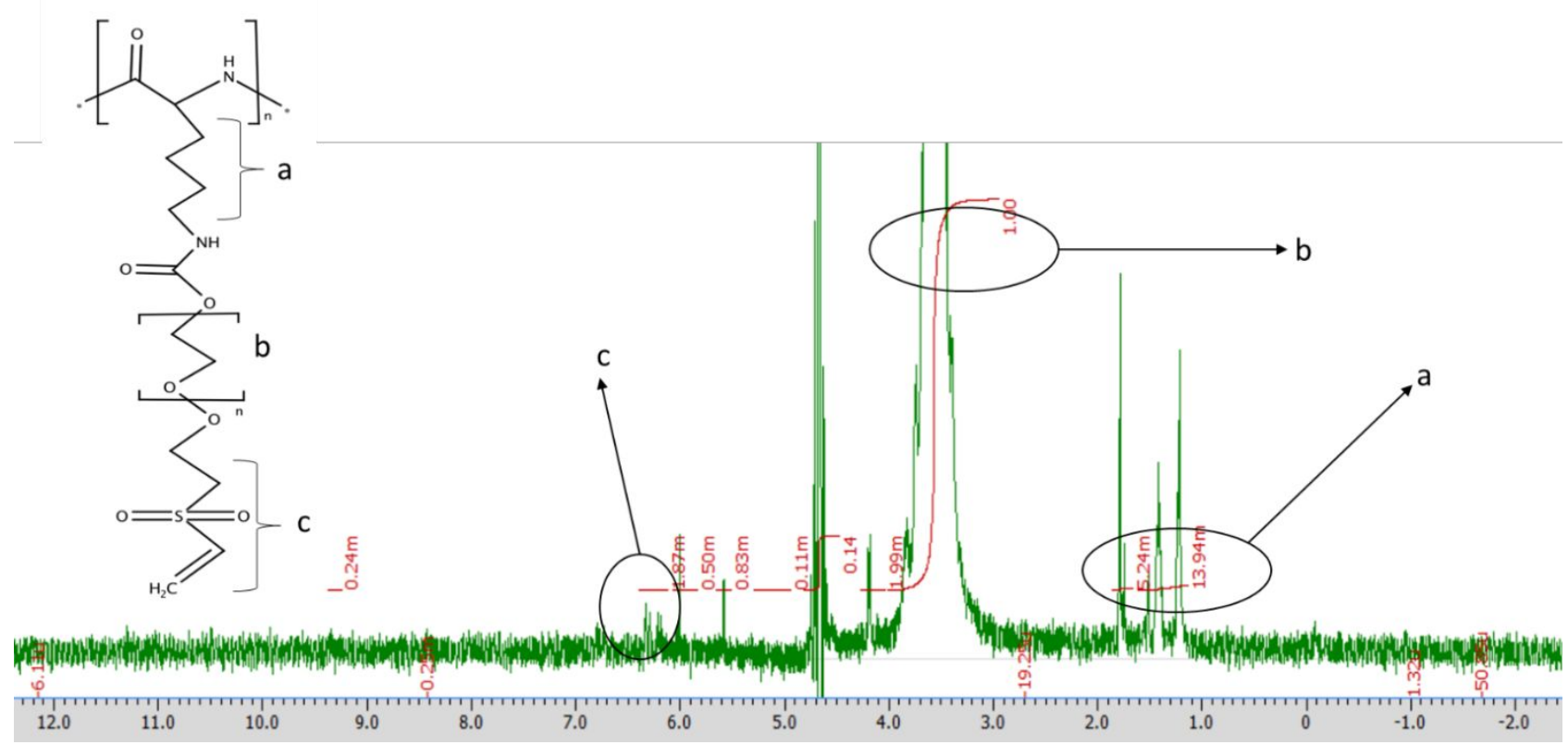

Figure S3. ${ }^{1} H$ NMR of the VS-PEG-PLL macromer formed.

S8 


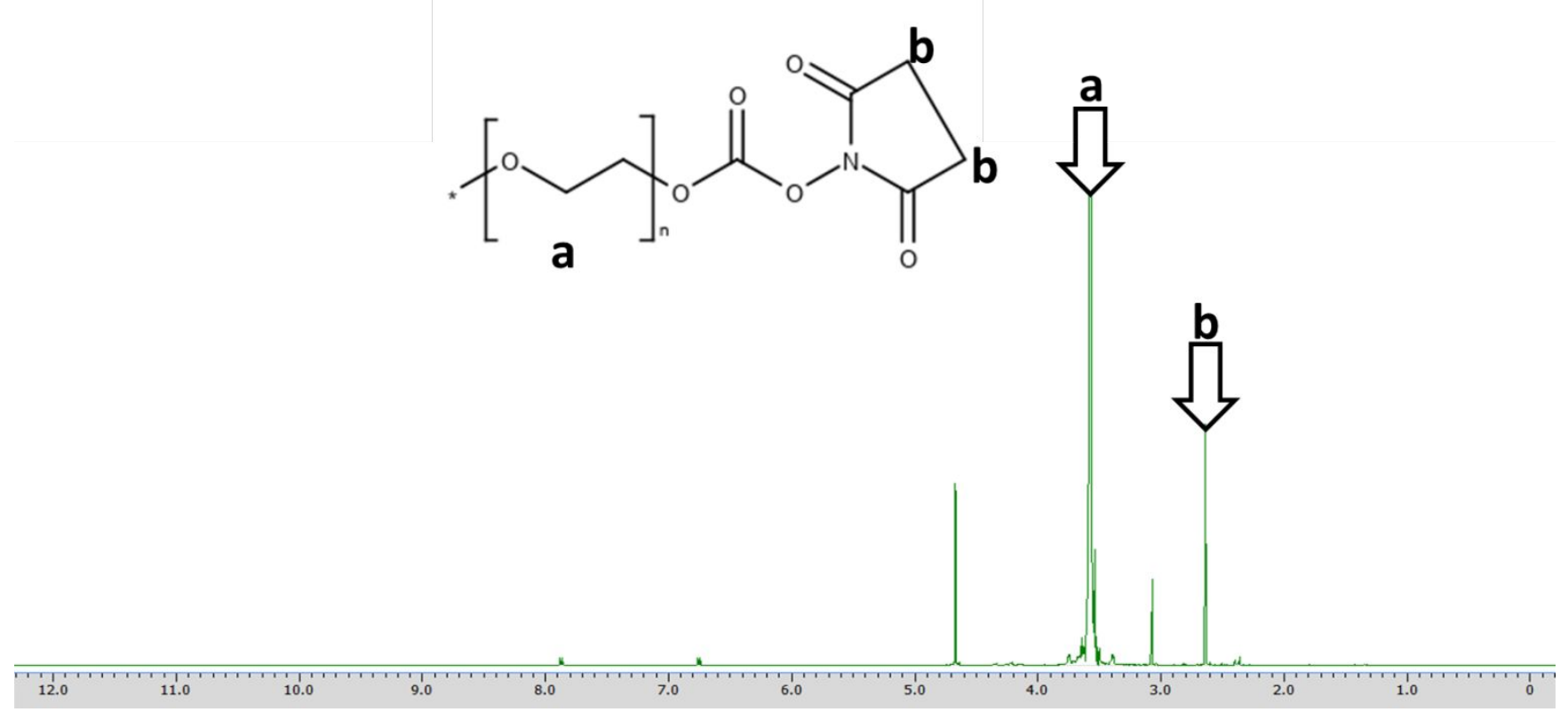

Figure S4. ${ }^{1} \mathrm{H} N \mathrm{NM}$ of the bifunctional PEG-SG (4.6kDa)

A

VS-SH-system

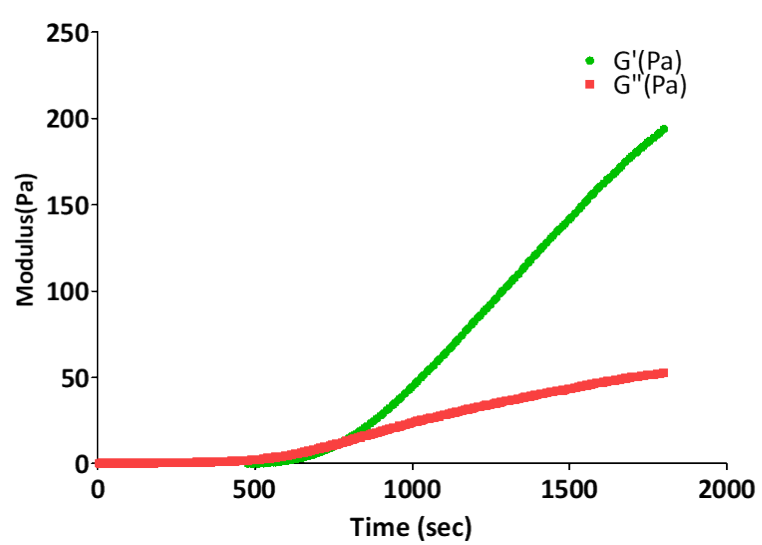

B

SG-NH2 system

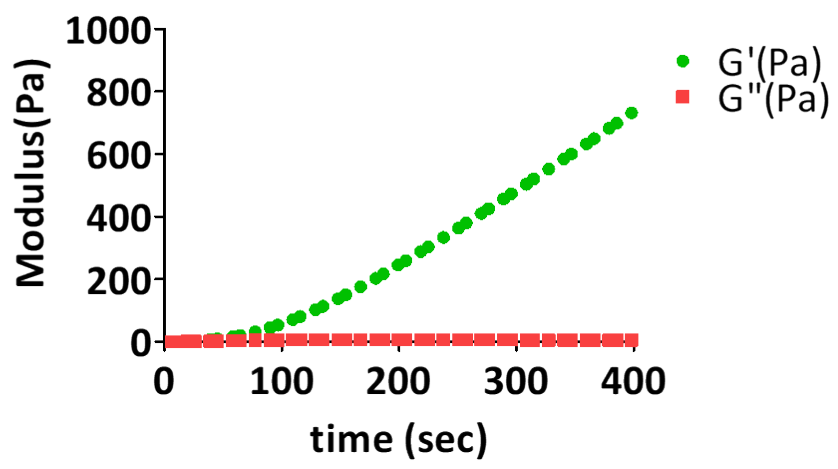

Figure S5. Time sweep of the a) VS-SH system and b) $\mathrm{SG}-\mathrm{NH}_{2}$ system showing the gelation time point where the Storage modulus $\left(G^{\prime}\right)$ is greater than Loss modulus $\left(G^{\prime}\right)$. This is the same data as in Figure 2b, but with the loss modulus information and a zoomed in version to see the gelation time clearly 


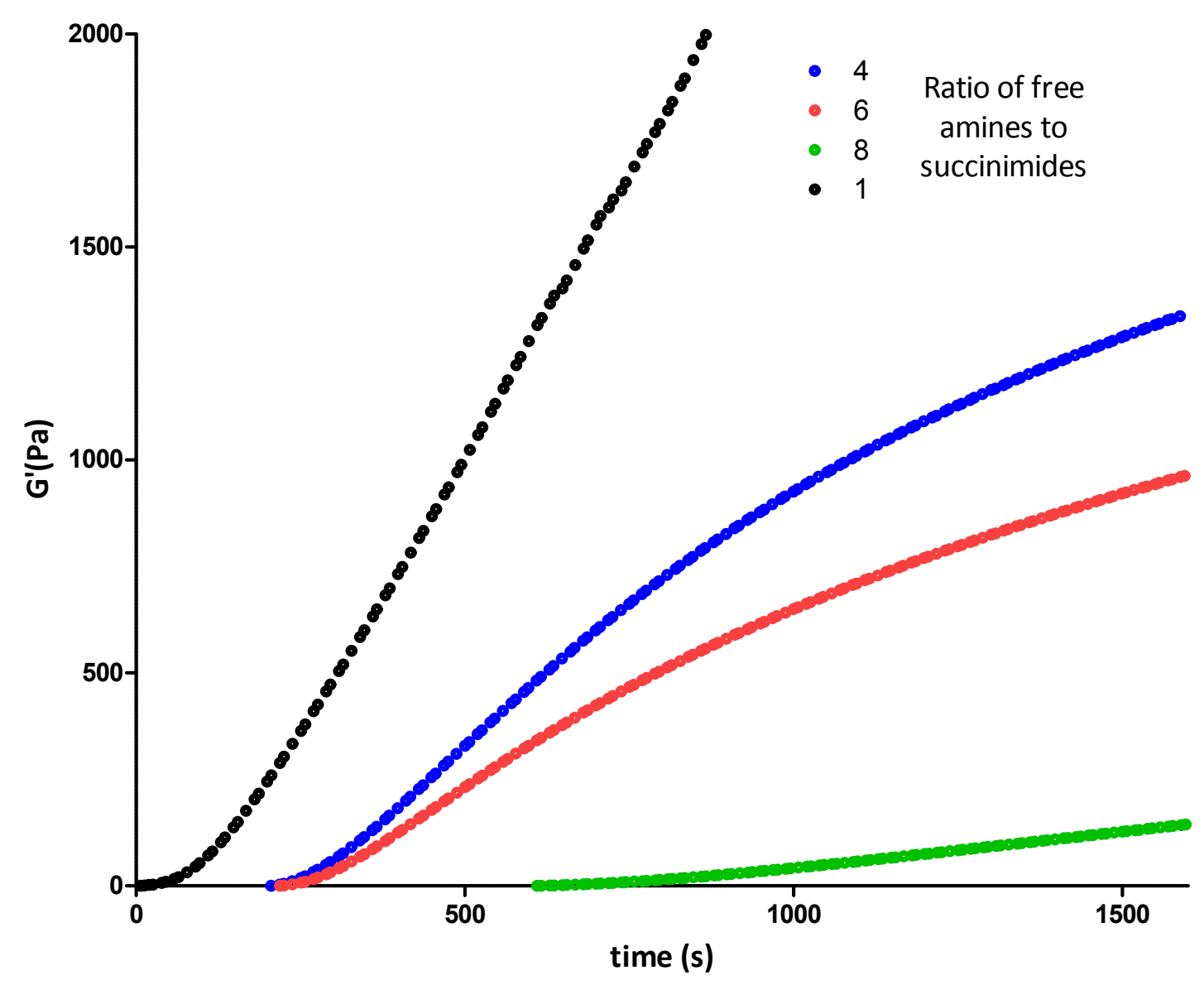

Figure S6. Time sweep of the succinimidyl amine hydrogel system by varying the ratio of free amines to succinimides, it can be noted that as the amount of cross linker is reduced, the gelation time decreases. 


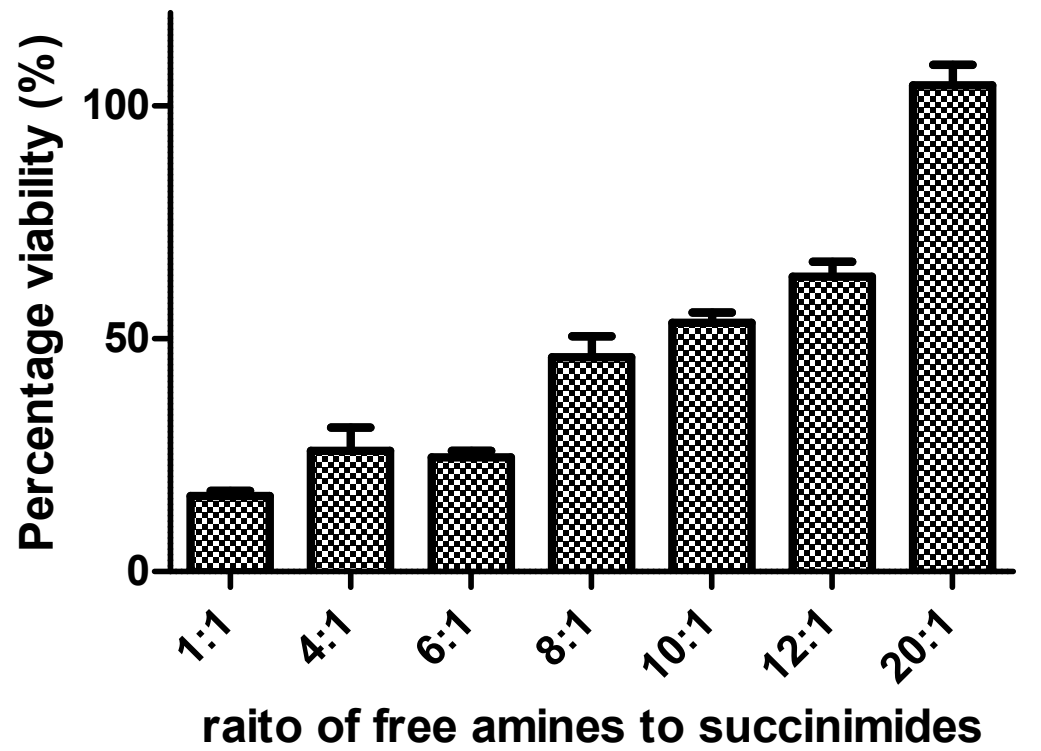

Figure S7. Viabilities of the A549 cells seeded in different PEG-PLL hydrogels synthesized by changing the amount of free amines to succinimides. 


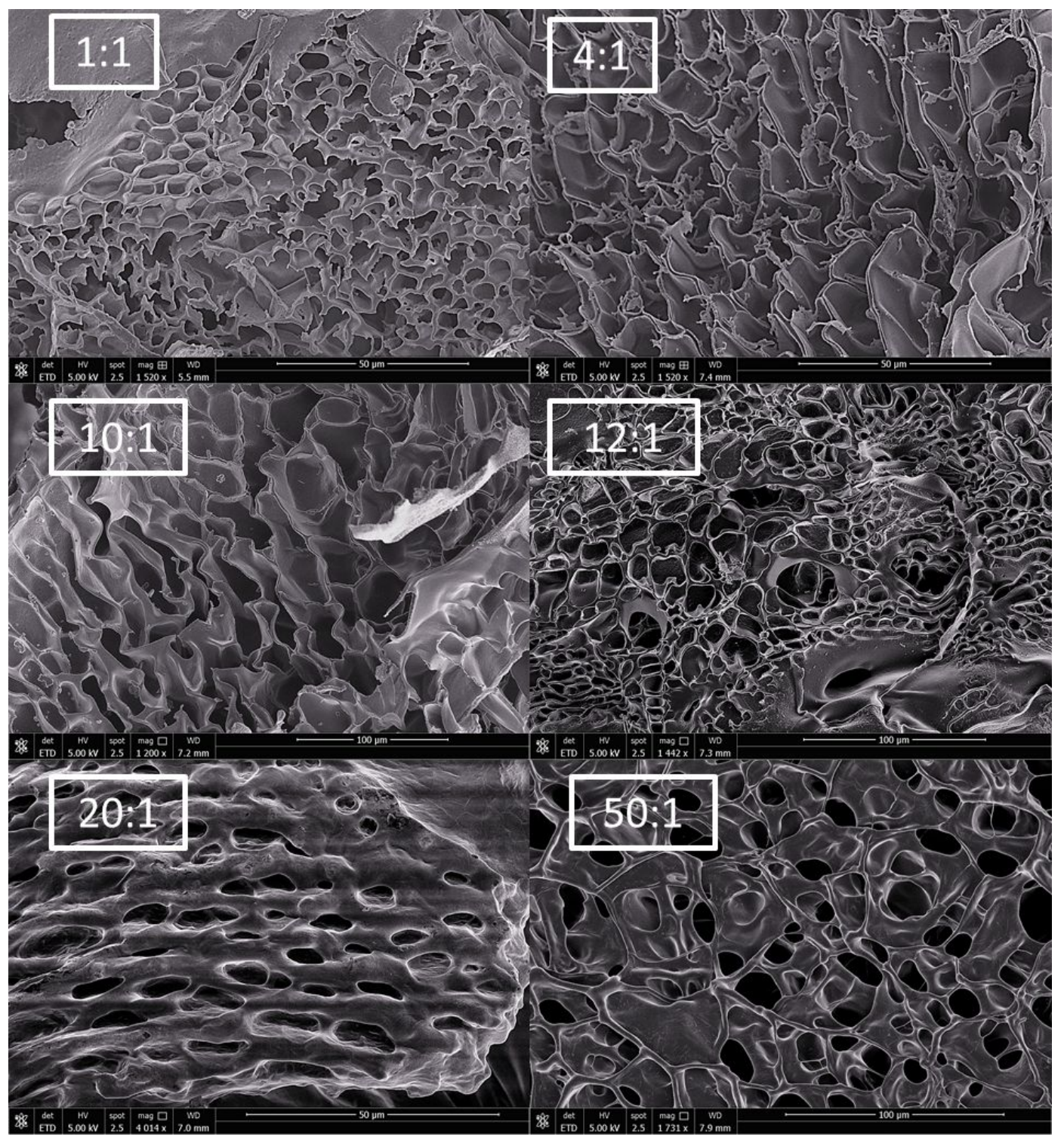

Figure S8. SEMs of different hydrogels synthesized using bifunctional PEG (4.6 kDa) and PLL (110 kDa) by altering the ratios of free amines to succinimides. 


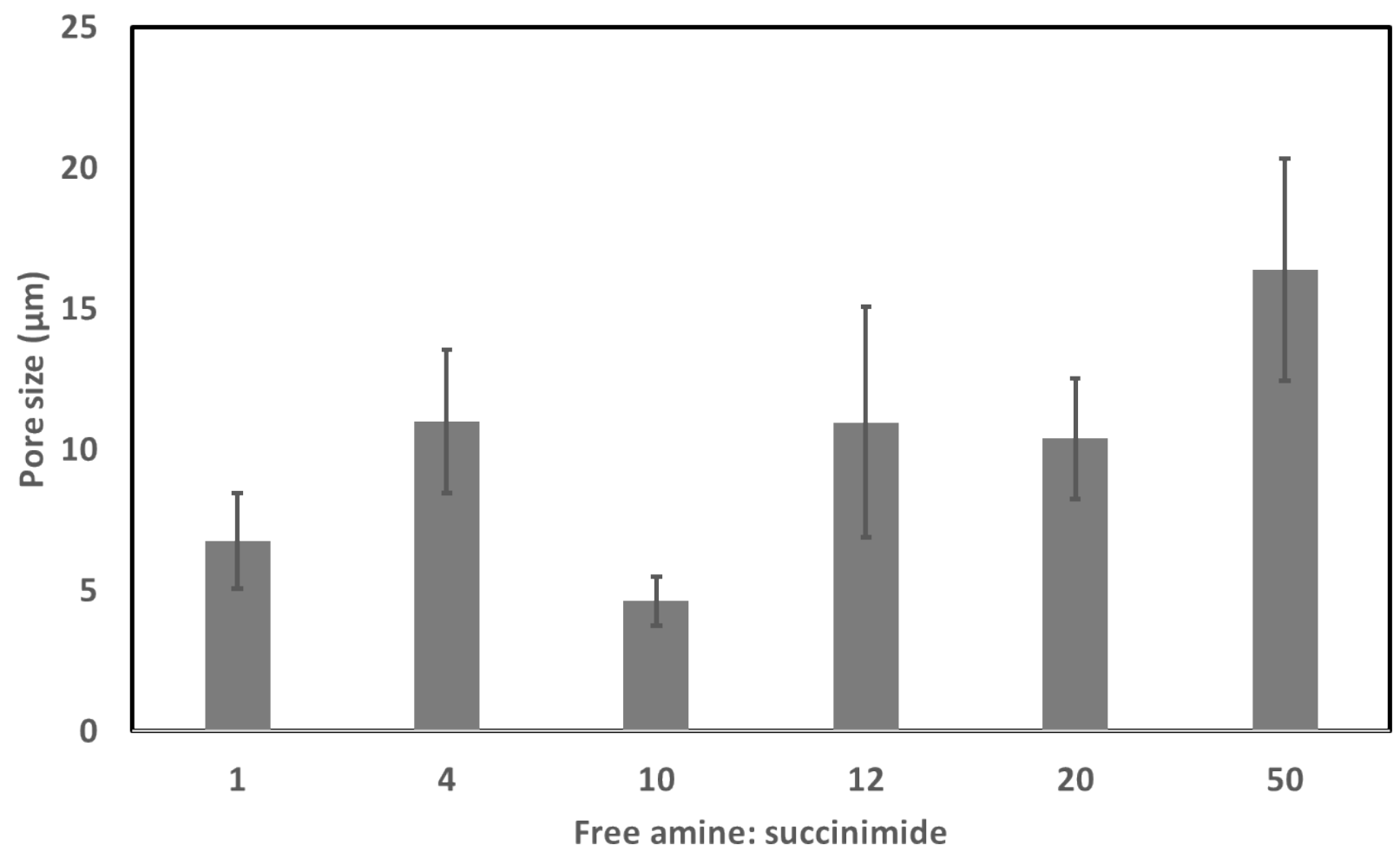

Figure S9. Pore size (diameter $=$ sqrt $(4 A / T)$ ) of the gels in $S G-N H 2$ gel library.

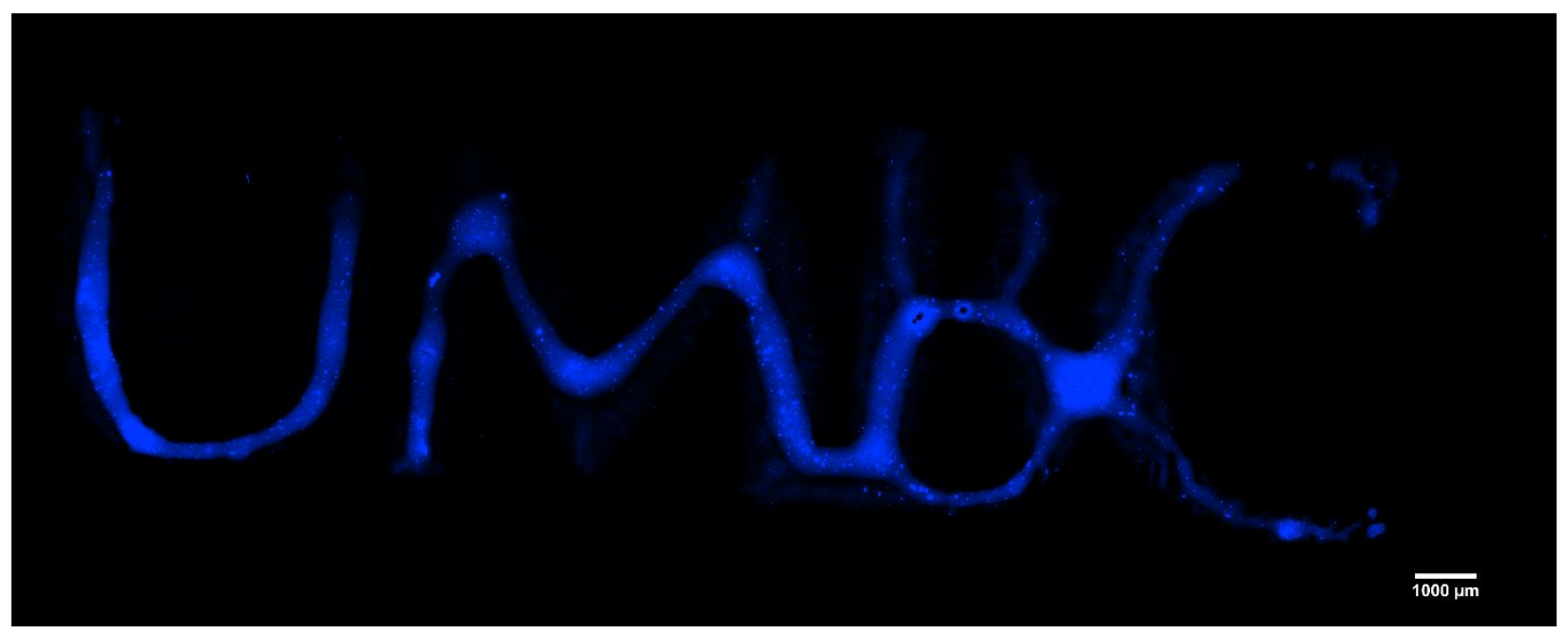

Figure S10. monolayered UMBC logo print, Florescent images of prints obtained using carboxylate microspheres embedded succinimidyl amine hydrogel as the bioink. The images are the composite images of a Z-stack of $15 X 15$ grid (Scale bar is $1 \mathrm{~mm}$ ) 

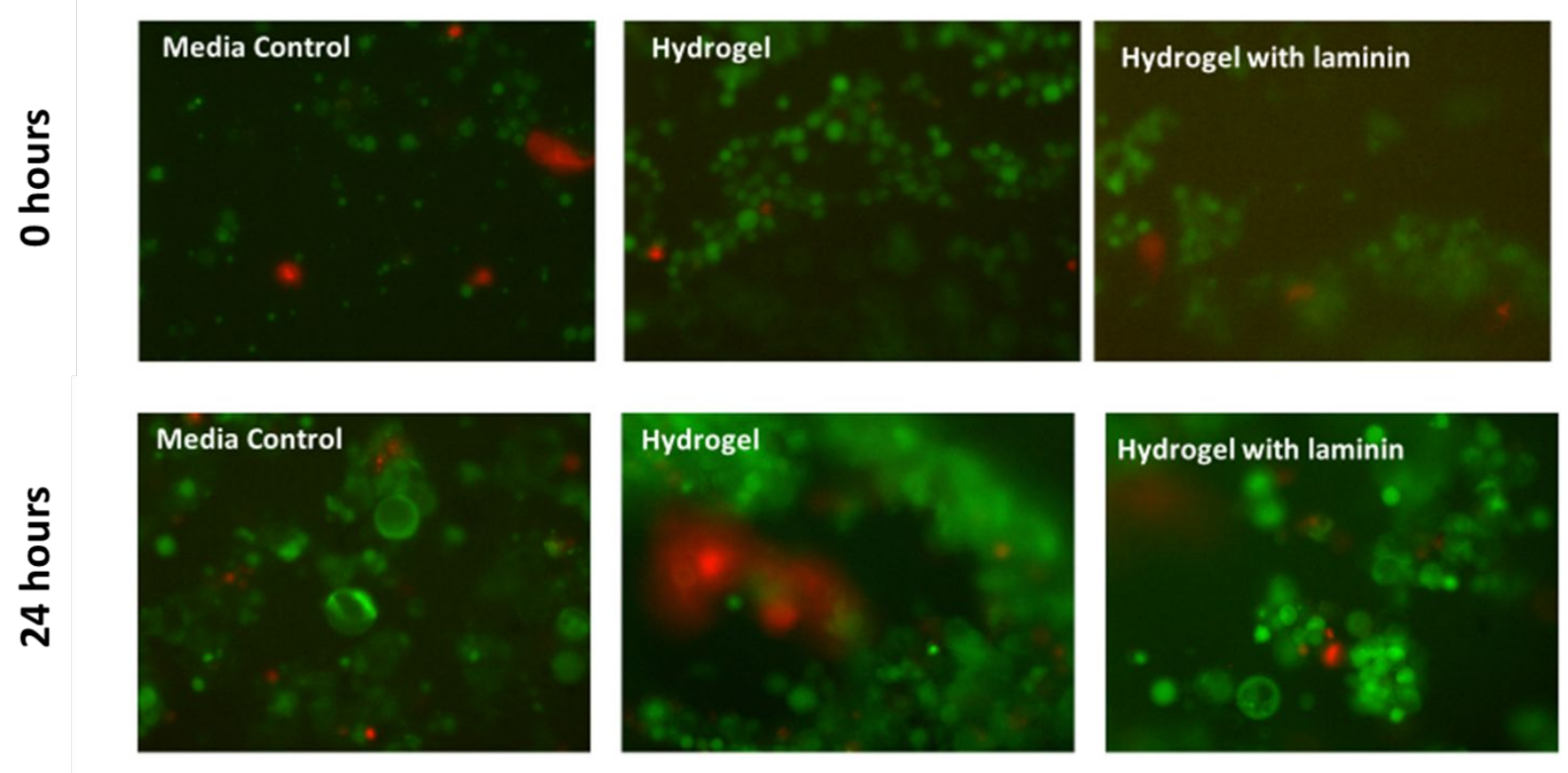

Figure S11. Live/Dead scans of the Caco-2 cells seeded on a PEG-PLL hydrogel base synthesized using 1:1 ratio of free amines to succinimides, PEG-PLL hydrogel base with 0.01\% laminin and well plate (media control) at $O$ hours and 24 hours after seeding the cells.

\section{REFERENCES}

1. Zuidema, J. M.; Rivet, C. J.; Gilbert, R. J.; Morrison, F. A., A protocol for rheological characterization of hydrogels for tissue engineering strategies. Journal of biomedical materials research. Part B, Applied biomaterials 2014, 102 (5), 1063-73.

2. Grotzky, A.; Manaka, Y.; Fornera, S.; Willeke, M.; Walde, P., Quantification of $\alpha-$ polylysine: a comparison of four UV/Vis spectrophotometric methods. Analytical Methods 2010, 2(10), 1448-1455.

3. Pandala, N.; Haywood, S.; LaScola, M. A.; Day, A.; Leckron, J.; Lavik, E., Screen Printing to Create 3D Tissue Models. ACS Applied Bio Materials 2020. 
